\title{
NEW RESULTS ON THE EXISTENCE OF THE GENERALIZED PARETO EQUILIBRIUM
}

\author{
MONICA PATRICHE \\ University of Bucharest, Faculty of Mathematics and Computer Science \\ 14 Academiei Street, Bucharest, Romania \\ E-mail: monica.patriche@yahoo.com
}

\begin{abstract}
In this paper, we state a new fixed point theorem for correspondences defined on Hausdorff locally convex spaces and we use it to prove the existence of the generalized weighted Nash equilibrium and the generalized Pareto equilibrium of a constrained multi-criteria game.

Key Words and Phrases: fixed point theorem, generalized weighted Nash equilibrium, generalized Pareto equilibrium, generalized multiobjective game.
\end{abstract}

2010 Mathematics Subject Classification: 47H10, 91A47, 91A80.

Acknowledgements. The author thanks to Professor João Paulo Costa from the University of Coimbra for the fruitful discussions and for the hospitality he proved during the visit to his department.

The author also thanks an anonymous referee for his careful reading and helpful suggestions, which led to an improved presentation of the manuscript.

\section{REFERENCES}

[1] P. Borm, F. Megen, S. Tijs, A perfectness concept for multicriteria games, Math. Meth. Oper. Res., 49(1999), 401-412.

[2] S. Chebbi, Existence of Pareto equilibria for non-compact constrained multi-criteria games, J. Appl. Anal., 14(2008), no. 2, 219-226.

[3] R.A. Dana, C. Le Van, Overlapping sets of priors and the existence of efficient allocations and equilibria for risk measures, Mathematical Finance, 20(2010), 327-339.

[4] X.P. Ding, Existence of Pareto equilibria for constrained multiobjective games in H-space, Computers \& Mathematics with Applications, 39(2000), 125-134.

[5] X.P. Ding, Pareto equilibria for generalized constrained multiobjective games in FC-spaces without local convexity structure, Nonlinear Anal., 71(2009), 5229-5237.

[6] K. Fan, A generalization of Tychonoff's fixed point theorem, Math. Ann., 142(1961), 305-310.

[7] D. Heath, H. Ku, Pareto Equilibria with coherent measures of risk, Mathematical Finance, 14(2004), 163-172.

[8] C.J. Himmelberg, Fixed points of compact multifunctions, J. Math. Anal. Appl., 38(1972), 205-207.

[9] W.K. Kim, Weight Nash equilibria for generalized multiobjective games, J. Chungcheong Math. Soc., 13(2000), 13-20.

[10] W.K. Kim, X.P. Ding, On generalized weight Nash equilibria for generalized multiobjective games, J. Korean Math. Soc., 40(2003), 883-899.

[11] E. Klein, A. Thompson, Theory of Correspondences, J. Wiley \& Sons, New York, 1984. 
[12] J.F. Nash, Non-cooperative games, Ann. Math., 54(1951), 286-295.

[13] M. Patriche, Equilibrium in Games and Competitive Economies, The Publishing House of the Romanian Academy, Bucharest, 2011.

[14] M. Patriche, Existence of equilibrium for multiobjective games in abstract convex spaces, Mathematical Reports, 16(2014), 243-252.

[15] M. Voorneveld, S. Grahn, M. Dufwenberg, Ideal equilibria in noncooperative multicriteria games, Math. Meth. Oper. Res., 52(2000), 65-77.

[16] S.Y. Wang, Existence of a Pareto equilibrium, J. Optim. Theory Appl., 79(1993), 373-384.

[17] H. Yu, Weak Pareto equilibria for multiobjective constrained games, Appl. Math. Let., 16(2003), 773-776.

[18] J. Yu, G.X.Z. Yuan, The study of Pareto equilibria for multiobjective games by fixed point and Ky Fan minimax inequality methods, Computers Math. with Applications, 35(1998), no. 9, $17-24$.

[19] X.Z. Yuan, The Study of Minimax Inequalities and Applications to Economies and Variational Inequalities, Memoirs of the Amer. Math. Soc., 132, 1998

[20] X.Z. Yuan, E. Tarafdar, Non-compact Pareto equilibria for multiobjective games, J. Math. Anal. Appl., 204(1996), 156-163.

[21] M. Zeleny, Game with multiple payoffs, Internat. J. Game Theory, 4(1976), 179-191.

Received: February 6, 2014; Accepted: October 26, 2014. 